\title{
An Algebraic Approach to the Planar Coloring Problem
}

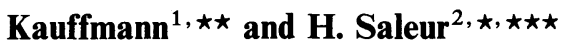 \\ 1 Department of Mathematics, Statistics and Computer Science, \\ University of Illinois at Chicago, Chicago, IL 60680, USA \\ 2 Department of Physics, Yale University, New Haven, CT 06511, USA
}

Received June 10, 1992

\begin{abstract}
We point out a general relationship between the planar coloring problem with $Q$ colors and the Temperley-Lieb algebra with parameter $\sqrt{Q}$. This allows us to give a complete algebraic reformulation of the four color result, and to give algebraic interpretations of various other aspects of planar colorings.
\end{abstract}

\section{Introduction}

The purpose of this paper is to delineate the relationship of the Temperley-Lieb algebra [TL] with planar graph coloring problems. The main result is a complete algebraic reformulation of the four color theorem $[\mathrm{AH}]$. This reformulation is a special case of a simply stated and more general conjecture about the TemperleyLieb algebra.

The paper is organized as follows. In the first section we recall the definition of the chromatic polynomial, the dichromatic polynomial, and of the Potts model. In the second section we recall the definition of the Temperley-Lieb algebra and of the Potts model representation. In Sect. Three we prove our first non-trivial result (Proposition 3.1). It states that the Potts model partition function (hence in particular the chromatic polynomial) for any planar graph can be written as the trace of a "transfer matrix" (Definition 3.2), a well defined product of elementary edge operators (Definition 2.3) in the Temperley-Lieb algebra. Such a result was known so far for regular lattices only. In Sect. Four we discuss some properties of the transfer matrices. We show in particular the reciprocal of Proposition 3.1 (Proposition 4.1), namely that an arbitrary product of edge operators can be

$\star$ On leave from SphT, Cen Saclay, F-91191 Gif Sur Yvette Cedex, France

$\star \star$ Work supported in part by NSF Grant \# DMS-882602, the program for Mathematics and Molecular Biology, UC Berkeley, and a visiting fellowship of the Japan Society for the promotion of science at Kyoto University, Kyoto, Japan

$\star \star \star$ Work supported in part by DOE Contact \# DE-AC02-76ERO3075 and by a Packard Fellowship for Science and Engineering 
considered as the transfer matrix for the Potts model on some planar graph. Restricting to the coloring problem we therefore have found that chromatic polynomials of planar graphs occur as traces of fully characterized products of operators in the Potts model representation of the Temperley-Lieb algebra, and conversely the trace of any such product is the chromatic polynomial of some planar graph. The situation compares favorably to the still open problem of characterization of chromatic polynomials [ST]. We also establish some connection between the existence of a transfer matrix that is a product of local edge operators and planarity (Proposition 4.2). Finally, we show how the noncolorability of a graph is expressed by the vanishing of the corresponding transfer matrix. In Sect. Five we consider the algebraic aspects of the planar coloring problem when the number of colors is smaller than four. We show in particular that the non-colorability of a set of simple graphs translates into the vanishing of symmetrizers $[\mathrm{Jo}, \mathrm{We}]$ in the Potts model representation of the Temperley-Lieb algebra (Proposition 5.3). Section Six deals with the algebraic reformulation of the planar coloring problem. Since the Temperley-Lieb algebra becomes simple for a number of colors greater or equal to four, the approach becomes independent of the Potts model representation and the four color result can be reformulated in a purely algebraic way (Theorem 6.4). In Sect. Seven we present an alternate formulation of these results in terms of the diagrammatic form of the TemperleyLieb algebra [Ka1] and a reformulation of the Potts model in terms of link diagrams. This gives alternate proofs for a number of our results, and provides an efficient language for translating between algebra and graph theory. The link diagrammatic approach opens the possibility of using knot theory in the study of these problems. Section Eight contains some speculations about the general properties of real zeroes of chromatic polynomials and the Beraha conjecture [Ba] from the point of view of the Temperley-Lieb algebra. The appendix discusses other (algebraic) reformulations of the four color problem in relation to this work.

While it has sometimes been said [D] that the four color problem is an isolated problem in mathematics, we have found that just the opposite is the case. The four color problem and the generalization discussed here is central to the intersection of algebra, topology and statistical mechanics. We hope that the work presented in this paper will stimulate more investigations of this fascinating structure.

We end this introduction with a brief statement of the Temperley-Lieb algebra, and our general conjecture. The reader will find this material repeated in the text, with appropriate context.

The Temperley-Lieb algebra (TL $)_{n}$ is an associative, non-commutative algebra of finite rank over the ring $\mathscr{R}=\mathbb{Q}[d]$, where $d$ is an algebraic variable commuting with all elements of (TL) ${ }_{n}$. We shall often specialize $d$ to be a specific real or complex number. ( $\mathbb{Q}$ denotes the rational numbers.) The multiplicative generators of $(\mathrm{TL})_{n}$ are denoted $1, e_{1}, e_{2}, \ldots, e_{n-1}$, and they satisfy the relations

$$
\begin{gathered}
e_{i}^{2}=d e_{i}, \quad i=1, \ldots, n-1, \\
e_{i} e_{i+1} e_{i}=e_{i}, \quad i=1, \ldots, n-2, \\
e_{i} e_{i-1} e_{i}=e_{i}, \quad i=2, \ldots, n-1, \\
e_{i} e_{j}=e_{j} e_{i}, \quad \text { if } \quad|i-j| \geqq 2 .
\end{gathered}
$$

Thus, a typical element of (TL) $)_{3}$ is of the form $a+b e_{1}+c e_{2}+d e_{1} e_{2}+f e_{2} e_{1}$. For example, $\left(e_{1} e_{2}\right)\left(e_{1} e_{2}\right)=\left(e_{1} e_{2} e_{1}\right) e_{2}=e_{1} e_{2}$ while $\left(e_{1} e_{2}\right)\left(e_{2} e_{1}\right)=\left(e_{1} e_{2} e_{2}\right) e_{1}=d e_{1} e_{2} e_{1}$ 
$=d e_{1}$. It is not hard to see that the rank of (TL) ${ }_{n}$ over $\mathscr{R}$ is equal to the Catalan number $C_{n}=\left(\begin{array}{c}2 n \\ n\end{array}\right) /(n+1)$, where $\left(\begin{array}{l}a \\ b\end{array}\right)=\frac{a !}{a !(a-b) !}$.

Our conjecture takes the following form. Let $d=2$, thus $e_{i}^{2}=2 e_{i}$ for all $i$. Let $R_{i}=1-\frac{e_{i}}{2}$ and $\bar{R}_{i}=-\frac{1}{2}+e_{i}$. Now note the following identities:

$$
\begin{gathered}
R_{i} e_{i}=\left(1-\frac{e_{i}}{2}\right) e_{i}=e_{i}-\frac{e_{i}^{2}}{2}=0, \\
e_{i \pm 1} \bar{R}_{i} e_{i \pm 1}=e_{i \pm 1}\left(e_{i}-\frac{1}{2}\right) e_{i \pm 1} \\
=e_{i \pm 1} e_{i} e_{i \pm 1}-\frac{e_{i \pm 1}^{2}}{2}=e_{i \pm 1}-e_{i \pm 1}=0 .
\end{gathered}
$$

Thus we have that

$$
R_{i} e_{i}=e_{i} R_{i}=0 \quad \forall i, \quad e_{i \pm 1} \bar{R}_{i} e_{i \pm 1}=0 \quad \forall i .
$$

Conjecture. Consider a product $\omega$ of the elements in Temperley-Lieb algebra $(\mathrm{TL})_{n}$ $(d=2): R_{i}, \bar{R}_{j}, e_{k}$. $\omega$ is equal to zero in (TL) if and only if $\omega$ is exterior equivalent to a product containing $R_{i} e_{i}$ or $e_{i \pm 1} \bar{R}_{i} e_{i \pm 1}$. (Exterior equivalence is generated by the multiplicative relations in Temperley-Lieb plus the relations $e_{i} R_{j}=R_{j} e_{i}, e_{i} \bar{R}_{j}$ $=\bar{R}_{j} e_{i}, R_{i} R_{j}=R_{j} R_{i}$ for $|i-j| \geqq 2$ or $|i-j|=0$.)

This conjecture implies the four color theorem. The four color theorem is itself equivalent to a specialization of this conjecture (see Theorem 6.4). The interested reader may enjoy exploring this conjecture on the purely algebraic grounds indicated here.

\section{The Potts Model and the Chromatic Polynomial}

A graph $G$ is a set of vertices, and edges joining pairs of vertices. We suppose without loss of generality that $G$ has no loop nor multiple edges. A vertex will be denoted $v_{\alpha}$, and an edge $\langle\alpha \beta\rangle$ if its extremities are $v_{\alpha}$ and $v_{\beta}$. Two vertices that are extremities of the same edge are called neighbours.

A color $\sigma$ is an integer in the set $\{1,2, \ldots, Q\}$, where $Q$ is the number of colors. A Q-configuration of colors on $G$ is a mapping from the set of vertices to the above set of colors, the vertex $v_{\alpha}$ having color $\sigma_{\alpha}$. A (proper) Q-coloring of $G$ is a configuration such that neighbours have different colors. We recall the [SK].

Proposition 1.1. Introduce $P_{G}(Q)$ the number of $Q$ colorings of $G$. Then $P_{G}$ is a monic polynomial in $Q$ with integer coefficients and of degree $V_{G}$, the number of vertices of G. $P_{G}$ is called the chromatic polynomial of $G$.

The $Q$-state Potts model is a model of interacting spins in statistical mechanics [Ba]. In this model a weight $\left(\varepsilon_{\alpha \beta}\right)^{\delta_{\sigma_{\alpha}} \sigma_{\beta}}$ is associated with every edge for a given $Q$-configuration, where $\delta$ is the Kronecker symbol, $\delta_{\sigma \sigma^{\prime}}=1$ if $\sigma=\sigma^{\prime}, 0$ if $\sigma \neq \sigma^{\prime}$, and $\varepsilon_{\alpha \beta}$ is an edge dependent real number $0 \leqq \varepsilon_{\alpha \beta}<\infty$ called coupling. We define 
Definition 1.2. The partition function of the $Q$-state Potts model on a graph $G$ with coupling $\varepsilon_{\alpha \beta}$ for the edge $\langle\alpha \beta\rangle$ is the sum

$$
Z_{G}\left(Q,\left\{\varepsilon_{\alpha \beta}\right\}\right)=\sum_{Q \text { configurations edges }\langle\alpha \beta\rangle}\left(\varepsilon_{\alpha \beta}\right)^{\delta_{\sigma_{\alpha} \sigma_{\beta}}} .
$$

It involves $Q^{V_{G}}$ terms.

Proposition 1.3. $Z_{G}\left(Q,\left\{\varepsilon_{\alpha \beta}\right\}\right)$ can be computed recursively by the two formulas

2)

$$
\begin{gathered}
Z_{G}=Z_{G_{\alpha \beta}^{\prime}}+\left(\varepsilon_{\alpha \beta}-1\right) Z_{G_{\alpha \beta}^{\prime \prime}}, \\
Z_{\bullet \cup G}=Q Z_{G}, \\
Z_{\bullet}=Q .
\end{gathered}
$$

In the first formula the triple $\left(G, G_{\alpha \beta}^{\prime}, G_{\alpha \beta}^{\prime \prime}\right)$ (see Fig. 1) denotes a graph $G$, a graph $G_{\alpha \beta}^{\prime}$ obtained from $G$ by deleting the edge $\langle\alpha \beta\rangle$ (but retaining the end points of the edge) and a graph $G_{\alpha \beta}^{\prime \prime}$ obtained from $G$ by collapsing the same edge. In Eq. (2), the point denotes a graph with one vertex and no edges. The symbol $\cup$ denotes disjoint union.

In the case of a uniform set of couplings $\varepsilon_{\alpha \beta}=\varepsilon \forall\langle\alpha \beta\rangle, Z_{G}(Q, \varepsilon)$ equals the dichromatic polynomial of $G$. If $\varepsilon_{\alpha \beta}=0$, only $Q$ configurations with $\sigma_{\alpha} \neq \sigma_{\beta}$ contribute to the partition function. Hence

Proposition 1.4. $P_{G}(Q)=Z_{G}\left(Q, \varepsilon_{\alpha \beta}=0 \forall<\alpha \beta>\right)$.

The chromatic polynomial is thus a particular case of the $Q$-state Potts model partition function when all couplings are set equal to zero. (This is called the zero temperature antiferromagnetic limit in physics [Ba].)

If $\varepsilon_{\alpha \beta}=1$, the partition function is the same as the one of the graph with edge $\langle\alpha \beta\rangle$ removed.

Finally, we shall need in the following a regularized definition of the partition function when some of the couplings are infinite.

Definition 1.5. We set

$$
\begin{aligned}
& Z_{G}\left(Q, \varepsilon_{\alpha \beta}=\infty, 0 \leqq \varepsilon_{\gamma \delta}<\infty \text { otherwise }\right) \\
& \quad=\lim _{\varepsilon \rightarrow \infty} \varepsilon^{-1} Z_{G}\left(Q, \varepsilon_{\alpha \beta}=\varepsilon, 0 \leqq \varepsilon_{\gamma \delta}<\infty \text { otherwise }\right) .
\end{aligned}
$$

(The existence of the limit is obvious from the above definitions.)

The Definition 1.5 can be iterated to several infinite couplings. With this definition, only $Q$ configurations with $\sigma_{\alpha}=\sigma_{\beta}$ contribute to the partition function when $\varepsilon_{\alpha \beta}=\infty$.

In the following, rather than involve infinite couplings through a limit process, we shall simply define certain cases of infinite coupling by direct form. Thus we take $\varepsilon_{\alpha \beta}$ in $[0, \infty)$.
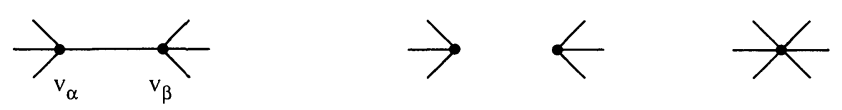

Fig. 1 


\section{Temperley-Lieb Algebra}

Define $r=\mathbb{C}^{Q}$ the linear span of colors $\sigma$ of a vertex $v$. In the vector space $R=r^{\otimes I} \mathrm{a}$ natural basis is provided by vectors $\underline{\sigma}=\left(\sigma_{1}, \sigma_{2}, \ldots, \sigma_{\mathrm{I}}\right)$, where $\sigma_{i}$ belongs to the color-set $\{1,2, \ldots, Q\}$.

Definition 2.1. In $R=r^{\otimes 1}$ we define $2 \mathfrak{l}-1$ square matrices of dimension $Q^{1} \times Q^{1}$ by their matrix elements

$$
\begin{gathered}
\left(e_{2 i-1}\right)_{\underline{\alpha \sigma^{\prime}}}=Q^{-1 / 2} \prod_{\substack{j=1 \\
j \neq i}}^{\mathrm{I}} \delta_{\sigma_{j} \sigma_{j}^{\prime}}, \\
\left(e_{2 i}\right)_{\underline{g \sigma^{\prime}}}=Q^{1 / 2} \delta_{\sigma_{i} \sigma_{i+1}} \prod_{j=1}^{\mathrm{I}} \delta_{\sigma_{j} \sigma_{j}^{\prime}} .
\end{gathered}
$$

with $i=1, \ldots$, l. As is well known one has [TL, Ba].

Proposition 2.2. The matrices $e_{i}$ satisfy the relations $[\mathrm{GHJ}]$

$$
\begin{gathered}
e_{i}^{2}=Q^{1 / 2} e_{i}, \\
e_{i} e_{i \pm 1} e_{i}=e_{i}, \\
{\left[e_{i}, e_{j}\right]=0 \text { for }|i-j| \geqq 2 .}
\end{gathered}
$$

The abstract algebra generated by the identity and $e_{i}$ 's, $i=1, \ldots, n-1$ obeying the relations of Proposition 2.2 is called the Temperley-Lieb algebra (TL) ${ }_{n}$. The matrices in Definition 2.1 provide a particular representation called the Potts model representation. We now introduce edge operators, where $\mathbf{1}$ is the identity in $R$.

Definition 2.3. We set

$$
\begin{gathered}
X_{2 i-1}(0 \leqq \varepsilon<\infty)=(\varepsilon-1) 1+Q^{1 / 2} e_{2 i-1}, \quad X_{2 i-1}(\infty)=1, \\
X_{2 i}(0 \leqq \varepsilon<\infty)=1+\frac{\varepsilon-1}{Q^{1 / 2}} e_{2 i}, \quad X_{2 i}(\infty)=\frac{e_{2 i}}{Q^{1 / 2}},
\end{gathered}
$$

$i=1, \ldots, \mathrm{I}$ and call $X$ 's edge operators.

Notice that $X_{2 i-1}(1)=Q^{1 / 2} e_{2 i-1}, X_{2 i}(1)=1$.

\section{The Transfer Matrices of Planar Graphs}

We now restrict to $G$ a planar graph. Edge operators can then be used to compute $Z_{G}$ as follows:

Proposition 3.1. Consider an arbitrary planar graph (which we suppose without loss of generality to have no loop nor multiple edges), drawn in the plane, all edges being straight lines $[\mathrm{F}]$. For any direction $\hat{x}$ in the plane (which we call time axis), there is a canonical matrix $\tau_{[G, \hat{x}]}$ called transfer matrix such that

- the partition function of the $Q$ state Potts model on $G$ with couplings $\varepsilon_{\alpha \beta}$ writes $Z_{G}=\operatorname{tr}\left(B \tau_{[G, \hat{x}]}\right)$,

- $\tau_{[G, \hat{x}]}$ is a fixed (independent of $Q$ and the couplings) product of edge operators $X_{i}\left(\varepsilon_{\alpha \beta}\right), X_{i}(0), X_{i}(\infty), i=1, \ldots, 2 \mathfrak{l}-1$ for some $\mathfrak{l}$,

- B is the product $Q^{1 / 2} \prod_{i=1}^{\mathfrak{l}} e_{2 i-1}$. 
This result was proven in the physics literature for $G$ a piece of regular lattice. We extend it here to the arbitrary case.

Proof. We draw $G$ in generic position with respect to the horizontal axis $\hat{x}$.

For each vertex $v_{\alpha}$ of $G$, draw through $v_{\alpha}$ a line parallel to the time axis. This line intersects some edges of $G$. At each intersection, introduce a new vertex called a projection of $v_{\alpha}$. In a given graph, if an edge is equipped with a designated interior point, then there is a unique way to obtain a new graph, identical to the old one except at the site of this edge. The edge is subdivided into two edges and the interior point becomes a common end point for the two new edges. In this way the set of projection vertices subdivides the original graph to a second graph. Augment this second graph further by introducing edges between successive projections of a given vertex. This gives a new graph $[G, \hat{x}]$.

Associate a coupling $\varepsilon=1$ to edges between successive projections of the same vertex (represented by a dotted line on figures). If $\langle\alpha \beta\rangle$ was an edge of $G$, and if by the above process some projected vertices $\gamma_{1}, \ldots, \gamma_{\kappa}$ are inserted in $\langle\alpha \beta\rangle$, then all the edges of $[G, \hat{x}]\left\langle\alpha \gamma_{1}\right\rangle\left\langle\gamma_{1} \gamma_{2}\right\rangle \ldots\left\langle\gamma_{\kappa} \beta\right\rangle$ are set to carry an infinite coupling (in the sense of Definition 1.5) except one that still carries $\varepsilon_{\alpha \beta}$ (which one does not matter). Infinite couplings are represented by heavy bonds on figures (see examples in Figs. 2 and 3).

These additional couplings clearly ensure $Z_{[G, \hat{x}]}=Z_{G}$.

A horizontal line of edges joining vertices of $[G, \hat{x}]$ is called a row. Horizontal edges are called equal space edges.

For $G$ in generic position, there are $V_{G}$ such rows. In practical situations it is useful to reduce this number by deformation of the graph that conserves its connectivities (see some examples in Fig. 3).

We set the final number of rows equal to 1 . On each row there are some vertices of $[G, \hat{x}]$. Call $t$ the maximum number of such vertices. We then number vertices on each row from left to right, the left-most having label one, the next label two while the right-most have several labels ... $t-1, t$ if the number of vertices on the considered row is less than $t$. These labels are called time coordinates.

G

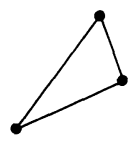$$
\hat{\mathrm{x}}
$$

(a)

G

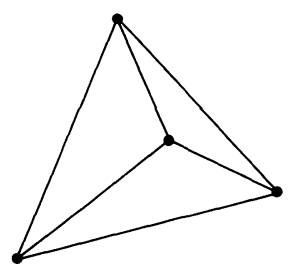

$[G, \hat{x}]$

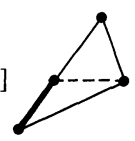

$[\mathrm{G}, \hat{\mathrm{x}}]$

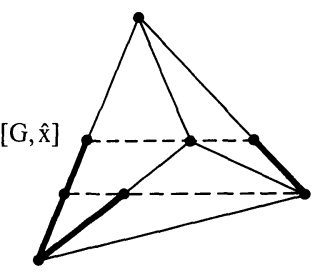




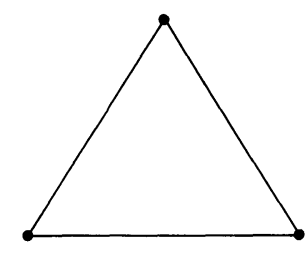

$\hat{\mathrm{x}}$

(a)
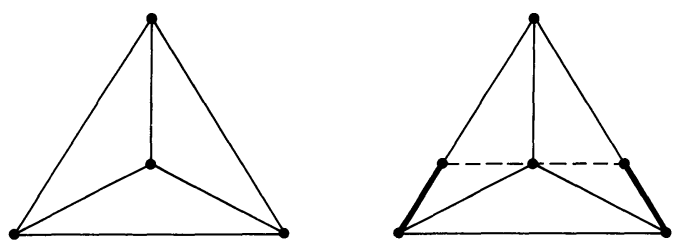

Fig. 3

(b)

A broken line of edges joining vertices of $[G, \hat{x}]$ with the same time coordinate is called a column. Edges joining vertices with the same time coordinate are called equal time edges. An edge that is neither equal space or equal time has its extremities belonging to successive rows and columns and is called a diagonal edge.

To each row we associate a copy of the space $r$. In the product $r^{\otimes I}$, spaces from left to right are associated with rows from top to bottom.

Definition 3.2. The transfer matrix of the Potts model on $G$ with couplings $\varepsilon_{\alpha \beta}$ for the edges $\langle\alpha \beta\rangle$ and a choice $\hat{x}$ of time axis is the matrix with entries

$$
\left(\tau_{[G, \hat{x}]}\right)_{\alpha, \sigma^{\prime}}=\sum_{Q \text { configurations edges }\langle\gamma \delta\rangle}\left(\varepsilon_{\gamma \delta} \delta^{\delta_{\sigma_{\nu}} \sigma_{\delta}},\right.
$$

where the sum is taken over $Q$ configurations such that vertices on the first column of $[G, \hat{x}]$ have fixed color $\sigma_{1} \ldots \sigma_{\mathrm{l}}$ and on the $t^{\text {th }}$ column $\sigma_{1}^{\prime} \ldots \sigma_{\mathrm{l}}^{\prime}$, the product over edges of $[G, \hat{x}]$, and the couplings are equal to some $\varepsilon_{\alpha \beta}$ if the corresponding edge belonged to $G$, one or infinity if the corresponding edge has been added in the above construction.

$\tau_{[G, \hat{x}]}$ can be built as a product of edge operators in the following way. With each column we associate the product

$$
\tau_{j}=\prod_{i=1}^{\mathrm{I}} X_{2 i}\left(\varepsilon_{\gamma \delta}\right)
$$

where $\varepsilon_{\gamma \delta}$ is the coupling associated with the edge $\langle\gamma \delta\rangle$ on the $j^{\text {th }}$ column, extremities belonging to the $i^{\text {th }}$ and $i+1^{\text {th }}$ row. With each pair of successive columns $j, j+1$ we associate a matrix $\tau_{j, j+1}$. To build $\tau_{j, j+1}$ consider first all vertices of the $j^{\text {th }}$ column that are not extremities of a diagonal edge between $j^{\text {th }}$ and $(j+1)^{\text {th }}$ columns. They are therefore connected to the $(j+1)^{\text {th }}$ column by an equal space edge $\langle\gamma \delta\rangle$ only, situated along the $i^{\text {th }}$ row. Form the product of $X_{2 i-1}$ operators for each of these edges. Then consider vertices $v_{\gamma}$ of the $j^{\text {th }}$ column that are extremities of a diagonal edge between $j^{\text {th }}$ and $(j+1)^{\text {th }}$ columns. Such an edge $\langle\gamma \delta\rangle$ has extremities $v_{\gamma}$ belonging to the $i^{\text {th }}$ row, $v_{\delta}$ to the $(i+1)^{\text {th }}$ row. Form the product of $X_{2 i}$ operators for each of these edges. Finally, vertices of the $j^{\text {th }}$ column 
that are an extremity of a diagonal edge between $j^{\text {th }}$ and $(j+1)^{\text {th }}$ columns may be also an extremity of an equal space edge between these. Form the product of $X_{2 i-1}$ operators for these edges (Fig. 4). Hence the form of $\tau_{j, j+1}$ is

$$
\tau_{j, j+1}=\left(\prod X_{2 i-1}\right)\left(\prod X_{2 i}\right)\left(\prod X_{2 i-1}\right) \text {. }
$$

It is then easy to check that

$$
\tau_{[G, \hat{x}]}=\tau_{1} \tau_{1,2} \tau_{2} \tau_{2,3} \ldots \tau_{t-1} \tau_{t-1, t} .
$$

The partition function of the Potts model itself is by definition

$$
Z_{G}=\sum_{\sigma \sigma^{\prime}}\left(\tau_{[G, \hat{x}]}\right)_{\sigma, \sigma^{\prime}}
$$

Using the formulas in Definition 2.3 this reads

$$
Z=\operatorname{Tr}\left(Q^{\mathrm{I} / 2} \prod_{i=1}^{1} e_{2 i-1} \tau_{[G, \hat{x}]}\right) .
$$

Example. The graph of Fig. 2a is in generic position, with $\mathrm{I}=3$. If all edges of $G$ carry a coupling $\varepsilon$ we get

$$
\begin{aligned}
\tau_{G} & =X_{2}(\varepsilon) X_{4}(\infty) X_{3}(1) X_{2}(\varepsilon) X_{4}(\varepsilon) \\
& =\left[x e_{4}+e_{4} e_{3}+\left(3 x^{2}+x^{3} Q^{1 / 2}\right) e_{2} e_{4}+x\left(e_{2} e_{4} e_{3}+e_{4} e_{3} e_{2}\right)\right] .
\end{aligned}
$$

For the graph in the position of Fig. $3 \mathrm{a}, \mathrm{I}$ is reduced to 2 and

$$
\tau_{G}=X_{2}(\varepsilon) X_{3}(\varepsilon) X_{2}(\varepsilon)=Q^{1 / 2}\left[x+\left(3 x^{2}+x^{3} Q^{1 / 2}\right) e_{2}+e_{3}+x\left(e_{2} e_{3}+e_{3} e_{2}\right)\right],
$$

where $x=(\varepsilon-1) / Q^{1 / 2}$. Hence in the first case we get the following expression for the partition function:

$$
Z=Q^{2} \operatorname{tr}\left[\left(1+2 x Q^{-1 / 2}\right) e_{4} e_{1} e_{3} e_{5}+\left(x+3 x^{2} Q^{-1 / 2}+x^{3}\right) e_{2} e_{4} e_{3} e_{1} e_{5}\right],
$$

and in the second case

$$
Z=Q^{3 / 2} \operatorname{tr}\left[\left(2 x+Q^{1 / 2}\right) e_{1} e_{3}+\left(x Q^{1 / 2}+3 x^{2}+x^{3} Q^{1 / 2}\right) e_{2} e_{1} e_{3}\right] .
$$

Traces of products of Temperley-Lieb matrices in the Potts model representation can be computed according to the following rules that define, for an arbitrary representation, the Jones trace [GHJ], denoted in general by Tr in the following
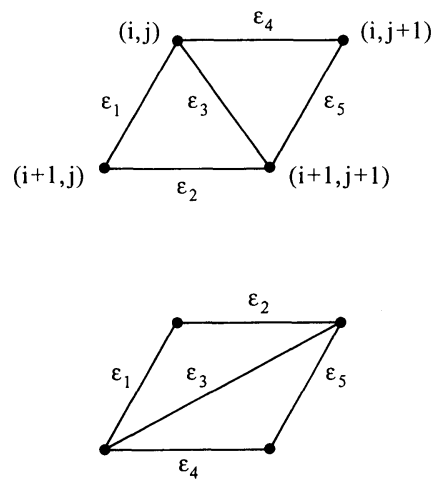


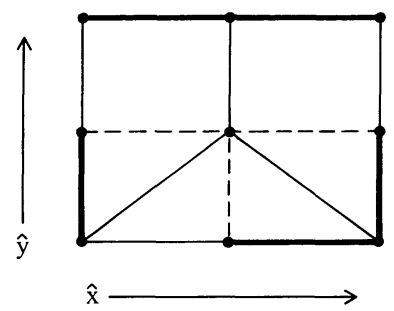

Fig. 5

\section{Proposition 3.3.}

$$
\begin{gathered}
\operatorname{tr} e_{i}=Q^{-1 / 2} \operatorname{tr} 1, \\
\operatorname{tr}\left[w\left(1, e_{1}, \ldots, e_{i-1}\right) e_{i}\right]=Q^{-1 / 2} \operatorname{tr} w\left(1, e_{1}, \ldots, e_{i-1}\right),
\end{gathered}
$$

where $w$ is any word on $1, e_{1}, \ldots, e_{i-1}$ and $\operatorname{tr} 1=\operatorname{dim} r=Q^{1}$.

Example. In the first case we get

$$
\begin{aligned}
Z & =Q^{2} Q^{3}\left(Q^{-2}+3 x Q^{-5 / 2}+3 x^{2} Q^{-3}+x^{3} Q^{-5 / 2}\right) \\
& =Q^{3}+3(\varepsilon-1) Q^{2}+3(\varepsilon-1)^{2} Q+(\varepsilon-1)^{3} Q,
\end{aligned}
$$

and in the second case

$$
Z=Q^{3 / 2} Q^{2}\left(Q^{-1 / 2}+3 x Q^{-1}+3 x^{2} Q^{-3 / 2}+x^{3} Q^{-1}\right)
$$

that coincides with the above.

Remarks. (i) It is clear that several extended graphs $[G, \hat{x}]$ can be associated to $G$ depending on the way it is drawn and the time axis that is chosen. One can also choose to suppress the edges that carry an $\varepsilon=1$ coupling as they do not modify the partition function. They are, however, useful in the construction of the transfer matrix $\tau$.

(ii) As a consequence of (i), several transfer matrices can be associated to the graph $G$. The question of their relations is open. But as we shall see later, this multiplicity does not cause a problem for our purposes.

(iii) Instead of $[G, \hat{x}]$ one can, by repeating the projection process along a chosen space axis $\hat{y}$, introduce a graph $[G, \hat{x}, \hat{y}]$ which is a piece of square lattice with some diagonals, such that $Z_{[G, \hat{x}, \hat{y}]}=Z_{G}$. Edges of $[G, \hat{x}, \hat{y}]$, as for $[G, \hat{x}]$, carry a coupling $\varepsilon_{\alpha \beta}, 1, \infty$, each $\varepsilon_{\alpha \beta}$ appearing once (see an example in Fig. 5).

\section{Some Properties of the Transfer Matrices}

Proposition 4.1. Consider any product $\tau$ of operators $X_{i}\left(\varepsilon_{\alpha \beta}\right)$, where the labels i take values $1, \ldots, 2 \mathrm{l}-1$ for some $\mathrm{l}$, the couplings $\varepsilon_{\alpha \beta}$ are a set of arbitrary numbers. Then there is a planar graph $G$ with as many edges as there are edge operators in $\tau$ and a time direction $\hat{x}$ for which $\tau$ is a transfer matrix $\tau_{[G, \hat{x}]}$ of the $Q$ state Potts model on $G$ with coupling $\varepsilon_{\alpha \beta}$ for every edge $\langle\alpha \beta\rangle$.

Proof. Draw I vertices and choose the time direction to be horizontal. Then read $\tau$ from left to right. Every time a $X_{2 i-1}\left(\varepsilon_{\alpha \beta}\right)$ operator is met, draw a new vertex further on the right on the $i^{\text {th }}$ row, and connect it to the preceding one with an edge 
that carries coupling $\varepsilon_{\alpha \beta}$. Every time a $X_{2 i}\left(\varepsilon_{\alpha \beta}\right)$ operator is met, simply add an edge carrying coupling $\varepsilon_{\alpha \beta}$ between the right-most vertices on $i^{\text {th }}$ and $(i+1)^{\text {th }}$ row. There is no obstruction to drawing the graph on the plane when an $X_{2 i-1}$ operator is met, since the rule is to extend the graph in a so far unoccupied region. To make sure there is no such obstruction with $X_{2 i}$ operators, notice that by definition edges have extremities that belong to equal or successive rows and equal or successive columns. Hence the only possible obstruction could be

But once the edge
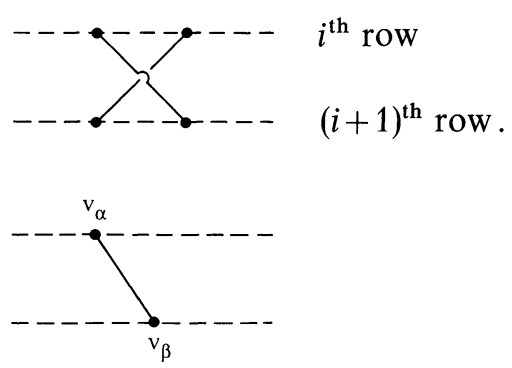

has been drawn, corresponding to some $X_{2 i}\left(\varepsilon_{\alpha \beta}\right)$ operator in the product, any other $X_{2 i}$ operator met later in the $\tau$ product has extremities coinciding with, or on the right of $v_{\alpha}, v_{\beta}$, so that the above obstruction is impossible.

Remarks. (i) In general the graph $G$ so obtained has multiple edges and loops. A loop ${ }^{2} \bigcirc$ contributes by a global factor $\varepsilon$ to the Potts model partition function. A multiple edge

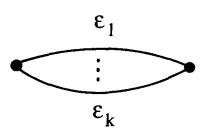

contributes as the single edge

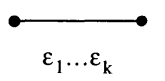

$G$ can thus be simplified to have neither loop nor multiple edges and still the same partition function (maybe up to a global scale factor).

(ii) If an infinite coupling appears in $\tau$, the corresponding edge can be retracted, if a coupling $\varepsilon=1$ appears, the corresponding edge can be erased, without changing the partition function.

Example. Loops can appear due to products $X_{2 i}(\varepsilon) X_{2 i}(\infty)$,

$$
\int \tau=X_{2}(\varepsilon) X_{2}(\infty)=\left(1+x e_{2}\right) \frac{e_{2}}{Q^{1 / 2}}=\frac{\varepsilon}{Q^{1 / 2}} e_{2} .
$$

Proposition 4.2. Consider an arbitrary graph $\mathscr{G}$. Suppose there is an $\mathrm{I}$ and a product $\tau_{\mathscr{G}}$ of edge operators $X_{i}\left(\varepsilon_{\alpha \beta}\right)$ (each edge of $\mathscr{G}$ appearing once). $X_{i}(1), X_{i}(\infty)$, $i=1, \ldots, 2 \mathrm{I}-1$ such that for any $Q$ and edge couplings $\varepsilon_{\alpha \beta}$ the partition function of the Potts model on $\mathscr{G}$ with couplings $\varepsilon_{\alpha \beta}$ can be written as $Z_{\mathscr{G}}=\operatorname{Tr}\left[\tau_{\mathscr{G}} Q^{1 / 2} \prod_{i=1}^{\mathrm{I}} e_{2 i-1}\right]$. Then $\mathscr{G}$ is planar. 
Proof. We can suppose without loss of generality that $\mathscr{G}$ has no loop nor multiple edges. Using Proposition 4.1 we can associate $\tau_{\mathscr{G}}$ to a planar graph $G^{\prime}$ and a time direction such that $\tau_{\mathscr{G}}$ is the transfer matrix of the Potts model with couplings $\varepsilon_{\alpha \beta}, 1, \infty$ on $G^{\prime}$. We can erase the edges carrying a coupling $\varepsilon=1$, contract the edges carrying $\varepsilon=\infty$ to get another planar graph $G$, satisfying

$$
Z_{\mathscr{G}}\left(Q,\left\{\varepsilon_{\alpha \beta}\right\}\right)=Z_{G}\left(Q,\left\{\varepsilon_{\alpha \beta}\right\}\right) .
$$

It is easy to see that $G$ has no loop nor multiple edges. Letting all $\varepsilon_{\alpha \beta}$ equal and go to zero, or all $\varepsilon_{\alpha \beta}$ equal and go to $\infty$ shows that the number of edges (vertices) of $\mathscr{G}$ and $G$ are equal

$$
E_{\mathscr{G}}=E_{G}, \quad V_{\mathscr{G}}=V_{G} .
$$

Suppose now $\mathscr{G}$ was non-planar. Then it contains a subgraph homeomorphic to $\Gamma_{5}$ or $\Gamma_{3,3}[\mathrm{SK}]$ :
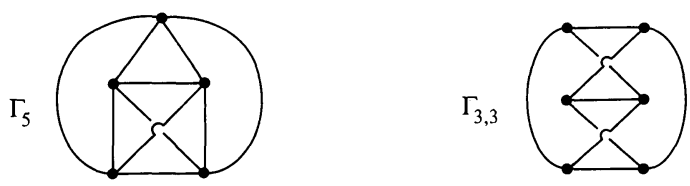

First suppose it contains $\Gamma_{5}$. Then choose the couplings $\varepsilon_{\alpha \beta}$ to be zero except for the edges of $\Gamma_{5}$. From (1) one deduces there is a planar subgraph $g$ of $G$ such that

and as before

$$
Z_{\Gamma_{5}}\left(Q,\left\{\varepsilon_{\alpha \beta}\right\}\right)=Z_{g}\left(Q,\left\{\varepsilon_{\alpha \beta}\right\}\right),
$$

$$
E_{g}=E_{\Gamma_{5}}=10, \quad V_{g}=V_{\Gamma_{5}}=5
$$

and $3 V_{g}-6=9$, while a necessary condition for planarity ( $g$ having no multiple edges) is $3 V_{g}-6 \geqq E_{g}[\mathrm{SK}]$. Hence this is impossible.

Suppose then $\mathscr{G}$ contains a subgraph homeomorphic to $\Gamma_{3,3}$. Again we can find a planar subgraph $g$ of $G$ such that

$$
Z_{\Gamma_{3,3}}\left(Q,\left\{\varepsilon_{\alpha \beta}\right\}\right)=Z_{g}\left(Q,\left\{\varepsilon_{\alpha \beta}\right\}\right) .
$$

We can expand these partition functions using Proposition 3.1. For $\mathscr{H}$ an arbitrary graph onto which a $Q$ state Potts model with coupling $\varepsilon$ for every edge is defined one finds easily

$$
Z_{\mathscr{H}}\left(Q,\left\{\varepsilon_{\alpha \beta}=\varepsilon\right\}\right)=\sum_{\mathscr{H}}(\varepsilon-1)^{E^{\prime}} Q^{C^{\prime}},
$$

where $\mathscr{H}^{\prime}$ are subgraphs of $\mathscr{H}$ made of a subset of $E^{\prime}$ of its edges and all its vertices grouped in $C^{\prime}$ connected components. Using Euler's relation

$$
V=E^{\prime}+C^{\prime}-L^{\prime}
$$

where $L^{\prime}$ is the number of independent circuits in $\mathscr{H}$, and setting $\varepsilon-1=\alpha Q$ we get

$$
Z_{\mathscr{H}}=\sum_{\mathscr{H}^{\prime}} \alpha^{E^{\prime}} Q^{V+L^{\prime}}
$$

$Q$ is now a formal variable that we let go to zero

$$
Q^{-V-1} Z_{\mathscr{H}} \cong \frac{1}{Q} \sum_{\text {no circuit }} \alpha^{E^{\prime}}+\sum_{\mathscr{H}^{\prime} \text { with one circuit }} \alpha^{E^{\prime}}+O(Q), \quad Q \rightarrow 0 ;
$$


by applying this to both sides of (2) we get

$$
\gamma_{\Gamma_{3,3}}=\gamma_{g}
$$

where $\gamma$ is the girth, length of the shortest circuit, that can be identified as the smallest $\alpha$ degree in the constant term of (3). Hence

and $\left[\frac{\gamma_{g}}{\gamma_{g}-2}\right]^{1} \begin{gathered}\gamma_{g}=\gamma_{\Gamma_{3,3}}=4, \quad E_{g}=E_{\Gamma_{3,3}}=5, \quad V_{g}=V_{\Gamma_{3,3}}=6, \\ \left.\left(V_{g}-2\right)=8 \text {, while a necessary condition for planarity [SK }\right] \text { ( } g \text { being }\end{gathered}$ obviously not a tree) is $\left[\frac{\gamma_{g}}{\gamma_{g}-2}\right]\left(V_{g}-2\right) \geqq E_{g}$.

We have again reached a contradiction. Therefore, $\mathscr{G}$ is planar.

Proposition 4.3. A planar graph $G$ is not $Q$-colorable if and only if the transfer matrix of the $Q$ state Potts model on $G$ with coupling $\varepsilon_{\alpha \beta}=0$ for every edge $\langle\alpha \beta\rangle$ is zero for some time axis. It then vanishes for any choice of time axis.

Proof. It follows from Definition 3.2.

\section{Algebraic Aspects of the Planar Coloring Problem for $Q<4$}

A transfer matrix of a graph $G$ that is non- $Q$-colorable can vanish via two mechanisms. The first is that $\tau_{G}$ can be zero in the Temperley-Lieb algebra itself. An example is the graph

$$
\int \tau_{G}=\left(1-\frac{e_{2}}{Q^{1 / 2}}\right) \frac{e_{2}}{Q^{1 / 2}}=0 \quad \forall Q .
$$

The second is that $\tau_{G}$ can be nonzero in the algebra, but vanish when evaluated in the Potts representation. An example is the graph

that is not $Q=1$ colorable and has $\tau_{G}=1-\frac{e_{2}}{Q^{1 / 2}}$. Hence $e_{2}(Q=1)=Q^{1 / 2}=1$ as can be checked using formulae in Definition 2.1. In this case, an extra relation among the Potts model Temperley-Lieb generators has to be satisfied in addition to those of Proposition 2.2.

We now recall

Definition 5.1. The symmetrizers [Jo, We] of order $n$ in the Temperley-Lieb algebra are defined recursively by

$$
S_{n}\left(e_{i}, \ldots, e_{i+n-2}\right)=S_{n-1}\left(e_{i}, \ldots, e_{i+n-3}\right)-t_{n} S_{n-1} e_{i+n-2} S_{n-1}, \quad n \geqq 2,
$$

where $S_{1}=1, t_{n}=\frac{1}{Q^{1 / 2}-t_{n-1}}, t_{1}=0$ and obey

$$
S_{n}^{2}=S_{n}, \quad S_{n} e_{j}=e_{j} S_{n}=0, \quad j=i, \ldots, i+n-2 .
$$

${ }^{1}[$ ]means integer part 
One has

Proposition 5.2. For $Q=1,2$ (resp. 3) the symmetrizers $S_{2}, S_{3}$ (resp. $S_{5}$ ) vanish in the Potts model representation of the Temperley-Lieb algebra.

Notice that due to Definition 5.1, if $S_{n}=0$ then $S_{n^{\prime}}=0, n^{\prime} \geqq n$. Hence for $Q=1$ for instance $S_{n}=0, n \geqq 2$.

Proof. This is easily checked by direct computation. One can also use the results of [SB] to decompose the Potts model representation onto the irreducible representations of the Temperley-Lieb algebra provided by interacting around face models and appeal to the results of [Jo, We].

We now point out how the corresponding extra relations among Potts model Temperley-Lieb generators for $Q=1,2,3$ have a coloring interpretation.

Proposition 5.3. For $Q=1,2,3$ the non-colorability of the graphs
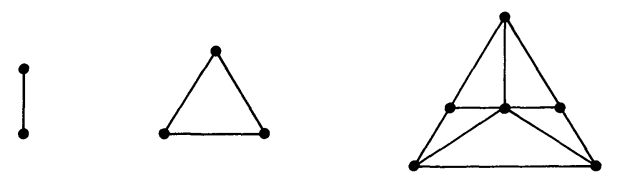

is expressed algebraically by the vanishing of $S_{2}, S_{3}, S_{5}$ in the Potts model representation.

Proof. The case $Q=1$ was considered above. For $Q=2$ and the graph

we have

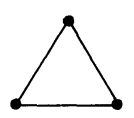

$$
\begin{aligned}
\tau & =X_{2}(0) X_{3}(0) X_{2}(0)=-1+\frac{2}{Q^{1 / 2}} e_{2}+Q^{1 / 2} e_{3}-e_{2} e_{3}-e_{3} e_{2} \\
& =-1+\sqrt{2}\left(e_{2}+e_{3}\right)-\left(e_{2} e_{3}+e_{3} e_{2}\right)=0 .
\end{aligned}
$$

By considering other orientations of $G$ we get

$$
1-\sqrt{2}\left(e_{i}+e_{i+1}\right)+\left(e_{i} e_{i+1}+e_{i+1} e_{i}\right)=0,
$$

which coincides with $S_{3}\left(e_{i}, e_{i+1}\right)=0$.

For $Q=3$ and the graph

one has

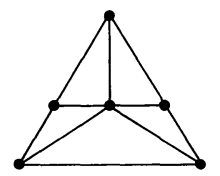

$$
\tau=X_{2}(0) X_{4}(0) X_{3}(0) X_{2}(0) X_{4}(0) X_{5}(0) X_{4}(0) X_{3}(0) X_{2}(0) X_{4}(0) \text {. }
$$

It is a long but straightforward exercise to check this coincides with $S_{5}\left(e_{2}, e_{3}, e_{4}, e_{5}\right)$ $=0$ for $Q=3$.

The vanishing of $\tau$ in the algebra itself can occur in less trivial cases than the loop. An example is 
Proposition 5.4. For $Q=1,2$ the non-colorability of the graph

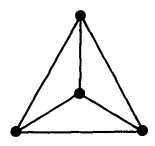

is expressed by the vanishing of $S_{2}, S_{3}$ in the Potts representation. But for $Q=3$ its transfer matrix is identically zero in the algebra itself.

Proof. One has

$$
\begin{aligned}
\tau & =X_{2}(0) X_{4}(\infty) X_{3}(1) X_{2}(0) X_{4}(0) X_{5}(0) X_{4}(0) X_{3}(1) X_{2}(0) X_{4}(\infty) \\
& =\frac{3-Q}{Q^{1 / 2}}\left[e_{4}-\frac{2}{Q^{1 / 2}} e_{2} e_{4}-Q^{1 / 2} e_{4} e_{3} e_{5} e_{4}+e_{2} e_{4} e_{3} e_{5} e_{4}+e_{4} e_{3} e_{5} e_{4} e_{2}\right] .
\end{aligned}
$$

\section{Algebraic Reformulation of the Planar Coloring Problem for $Q=4$}

In this algebraic setting $Q=4$ appears as a peculiar value: it is the first integer for which the Jones trace (Proposition 3.3) is faithful. More precisely, one has

Proposition 6.1 [GHJ]. For $Q \neq 4 \cos ^{2} \pi \frac{p}{n}$ with $\operatorname{gcd}(p, n)=1, n \geqq 2$, if $X$ is some nonzero operator in the Temperley-Lieb algebra, there exists another non-zero operator $Y$ in the Temperley-Lieb algebra such that $\operatorname{Tr} X Y \neq 0$, where $\operatorname{Tr}$ is the Jones trace computed according to the rules in (Proposition 3.3).

Suppose for $Q$ integer $\geqq 4$ that a transfer matrix of some graph was not zero in the algebra but zero in the Potts representation. Then for any $Y$ in the Potts representation, $\tau_{G} Y=0$, which implies $\operatorname{tr} \tau_{G} Y=0$. Now for the Potts representation the usual trace satisfies the Jones properties. Hence $\operatorname{Tr} \tau_{G} Y=0$ and from the above proposition we reach a contradiction. Thus

Proposition 6.2. For $Q$ integer $\geqq 4$ a planar graph $G$ is non- $Q$-colorable if and only if its transfer matrices $\tau_{G}$ vanish in the Temperley-Lieb algebra.

For $Q=4$, the edge operators that contribute to $\tau_{G}$ are $1, e_{2 i}, e_{2 i-1}$, $\bar{R}_{2 i-1}=-\frac{1}{2}+e_{2 i-1}, R_{2 i}=1-\frac{e_{2 i}}{2}$. We define

Definition 6.3. Two word products of $e_{i}, R_{j}, \bar{R}_{k}$, are exterior equivalent if they can be identified using relations

$$
e_{i}^{2}=2 e_{i}, \quad e_{i} e_{i \pm 1} e_{i}=e_{i}, \quad\left[X_{i}, Y_{j}\right]=0, \quad|i-j| \geqq 2 \quad \text { or } \quad i=j
$$

(but we do not use the explicit expressions of $R, \bar{R}$ ). In the last relation $X, Y$ are any of $e, R, \bar{R}$.

We can therefore reformulate the four color theorem $[\mathrm{AH}]$ as

Theorem 6.4. For any integer $n$, the only products of $1, e_{j}(j=1, \ldots, 2 n)$, $\bar{R}_{2 i-1}=-\frac{1}{2}+e_{2 i-1}, R_{2 i}=1-\frac{e_{2 i}}{2}(i=1, \ldots, n)$ that vanish in the Temperley-Lieb algebra $(\mathrm{TL})_{2 n+1}$,

$$
e_{i}^{2}=2 e_{i}, \quad e_{i} e_{i \pm 1} e_{i}=e_{i}, \quad\left[e_{i}, e_{j}\right]=0, \quad|i-j| \geqq 2
$$


are the ones that contain up to exterior equivalences as in Definition 6.3 one of the products $\left\{e_{2 j} R_{2 j}, e_{2 j} \bar{R}_{2 j \pm 1} e_{2 j}\right\}$ for some $j$.

We can reformulate the Temperley-Lieb relations for $Q=4$ by setting $t_{i}=1-e_{i}$. The $t_{i}$ satisfy then

$$
\begin{gathered}
t_{i}^{2}=\mathbf{1}, \\
t_{i} t_{i \pm 1} t_{i}=\mathbf{1}-t_{i}-t_{i \pm 1}+t_{i} t_{i \pm 1}+t_{i \pm 1} t_{i}, \quad i=1, \ldots, 2 n, \\
{\left[t_{i}, t_{j}\right]=0, \quad|i-j| \geqq 2 .}
\end{gathered}
$$

Of course, these relations are a quotient of the Weyl group relations. This is no surprise since for $Q=4$ the Temperley-Lieb algebra [which is associated to $U_{q} s l(2)$ for $Q$ generic, $Q^{1 / 2}=q+q^{-1}$ ] is the commutant of $s l(2)$ in the tensor product of fundamental representations. The definition of equivalence (Definition 6.2) immediately extends when using the $t_{i}$ 's. Alternatively, we can thus reformulate Theorem 6.4.

Corollary 6.5. Introduce in $\left(C^{2}\right)^{\otimes n}$ the transposition operators $t_{i}$. For integer $n$, the only products of $\mathbf{1}, \mathbf{1}-t_{j}(j=1, \ldots, 2 n), \bar{R}_{2 i-1}=\frac{1}{2}-t_{2 i-1}, R_{2 i}=\mathbf{1}+t_{2 i}(i=1, \ldots, n)$ that vanish are the ones containing one of $\left\{\left(1-t_{2 j}\right) R_{2 j},\left(1-t_{2 j}\right) R_{2 j \pm 1}\left(1-t_{2 j}\right)\right\}$ for some $j$ up to exterior equivalence.

From an algebraic point of view, the result without odd-even requirements for the $R$ indices looks as likely and leads to

Conjecture 1 . For any integer $n$, the only products of $1, e_{j}(j=1, \ldots, n), R_{i}, \bar{R}_{i}$ $(i=1, \ldots, n)$ that vanish in the Temperley-Lieb algebra (TL $)_{n+1}$ contain up to exterior equivalence one of $\left\{e_{j} R_{j}, e_{j} \bar{R}_{j+1} e_{j}\right\}$ for some $j$.

Of course, this conjecture implies Theorem 6.4.

\section{A Geometric Approach}

We now describe the reformulation of the dichromatic polynomial as a bracket polynomial on link diagrams. The bracket polynomial, $[K]$, in three variables $A$, $B$, and $d$ is defined as follows on a link diagram $K:[K]=\sum_{S}[K / S] d^{\|S\|}$. Here $S$ runs over all bracket states of the diagram $K,[K / S]$ is the product of the vertex weights of $S$ and $\|S\|$ is the number of circuits in the state $S$. A bracket state $S$ is obtained from the link diagram $K$ by replacing each crossing in the diagram by non-crossing segments in one of the two possible ways shown below:

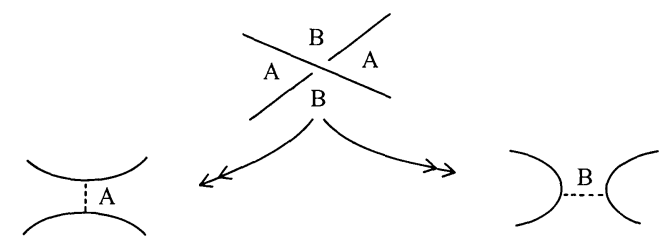


In this diagram, we have labelled pairs of regions at the crossing by the letters $A$ and $B$ so that the region to the left of an undercrossing line (as the observer faces the crossing) is always labelled $A$. The two splices then acquire the labels $A$ or $B$ according to the names of the regions joined by the splice. These labels are the vertex weights for the state. For example, if $K$ is the diagram

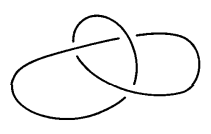

then the state $S$ shown below

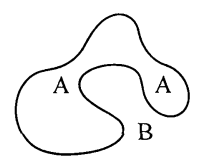

has vertex weights $A, A, B$ and only one circuit. Thus $[K / S]=A^{2} B$ and $\|S\|=d$.

For a simple example of a complete calculation of a bracket polynomial, let $K$ denote the diagram $\bigcirc$. Then there are two states for $K$ : A $\bigcirc$ B . Hence $[K]=A d+B d^{2}$. It is easy to see from the definition of the bracket polynomial that the following formulas are valid (note that $A, B$, and $d$ commute with one another):

$$
\begin{gathered}
{[\rtimes]=A[\asymp]+B[)(],} \\
{[O K]=d[K],} \\
{[O]=d .}
\end{gathered}
$$

Here the small diagrams stand for parts of otherwise identical larger diagrams. These formulas are analogs of the formulas we have already articulated for the dichromatic polynomial. Note that without further specialization of the variables $A, B, d$ the bracket is not a topological invariant of the link diagram. (See [Ka 1,2] for a discussion of specializations that yield the Jones polynomial.)

In [Ka 2] it is shown how the dichromatic polynomial for a planar graph can be written as a bracket evaluation. In order to recall this result, we need the notion of the alternating medial diagram $K(G)$ associated with a plane graph $G$. The alternating medial diagram $K(G)$ is obtained from $G$ by first placing a crossing on each edge of $G$ as shown below:

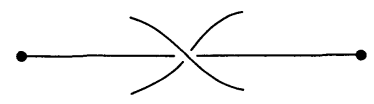

That is, the edge of $G$ goes through the $A$-regions of the crossing. The ends of these crossings are then connected to each other by the procedure illustrated below:

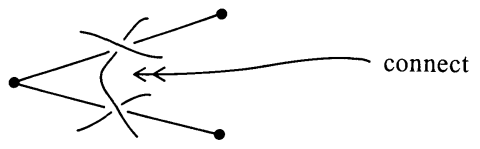


In other words, a free end at a crossing is connected to the nearest free end in the neighborhood of the vertex of that edge. For example:

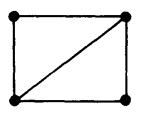

G
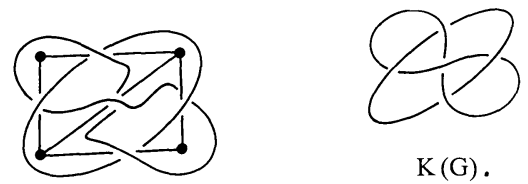

With this concept of alternating medial diagram in hand, we can state the

Theorem 7.1 [Ka 2]. Let $G$ be a plane graph, let $K(G)$ be the alternating medial for $G$. Let $\{K\}$ denote the bracket polynomial with $B=1, A=x\left(x=(\varepsilon-1) / Q^{1 / 2}\right), d=Q^{1 / 2}$. That is,

$$
\{K\}=[K] \quad\left(B=1, A=x, d=Q^{1 / 2}\right)
$$

so that

$$
\begin{gathered}
\{\varkappa\}=\{\rtimes\}+x\{\asymp\}, \\
\{O K\}=Q^{1 / 2}\{K\}, \\
\{O\}=Q^{1 / 2} .
\end{gathered}
$$

Then the dichromatic polynomial for $G$ is given by the formula

$$
Z_{G}(Q, \varepsilon)=Q^{V_{G} / 2}\{K(G)\},
$$

where $V_{G}$ denotes the number of vertices of $G$.

Example. $\{\infty\}=\{O O\}+x\{O\}=Q+x Q^{1 / 2}$

and

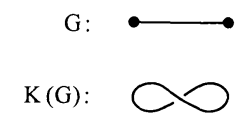

$$
Q^{V_{G} / 2}\{K(-)\}=Q^{2}+x Q^{3 / 2}=Q^{2}+Q(\varepsilon-1)=Z_{G} .
$$

For our purposes, the main point about this reformulation of the dichromatic polynomial is that it enables us to express $Z_{G}$ in terms of the Temperley-Lieb algebra for any plane graph $G$. This follows at once from a few remarks about the 3 -variable bracket. Let $K$ be any link diagram. Then we can arrange $K$ with respect to a height function so that it is a plat closure of an element in the braid monoid $\widetilde{B}_{n}$ (for some even integer $n$ ) where $\widetilde{B}_{n}$ is generated by the usual braiding generators

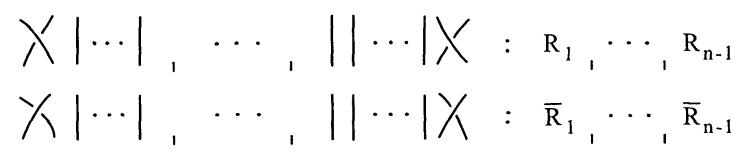

and the Temperley-Lieb generators

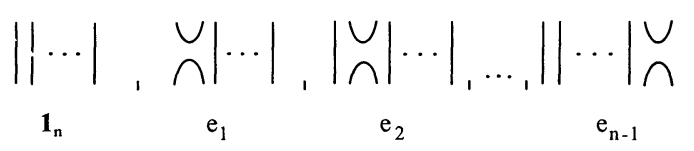


We have denoted the braiding generators by $R_{i}, \bar{R}_{i}$ rather than the usual notation $\left(\sigma_{i}, \sigma_{i}^{-1}\right)$ since we are not, at the diagrammatic level, assuming any further relations among them. Elements of the monoid $\widetilde{B}_{n}$ are multiplied just like braids, but diagrams are equivalent only up to graphical isotopy (where the crossings are regarded as 4-valent vertices with special structure). Natural relations ensue among the $e_{i}$ 's with the closed loop $O$ having value $d$ :

$$
\begin{aligned}
& \begin{array}{ll}
e_{i}^{2}=d e_{i} & \cup \\
& \bigcap
\end{array}=O \bigcap
\end{aligned}
$$

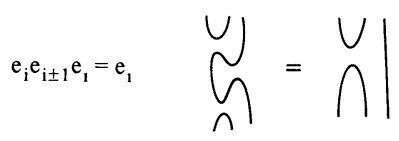

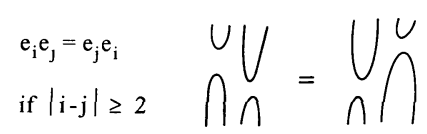

The $e_{i}$ 's represent paired maxima and minima. A typical plat closure has the form

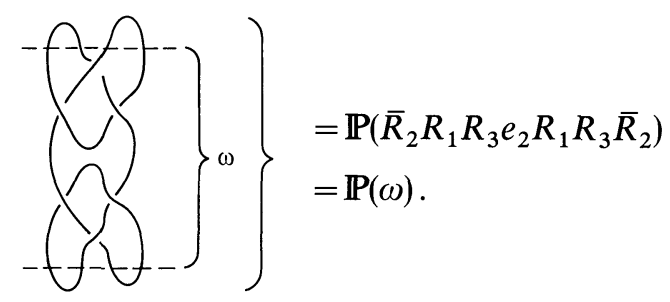

The word $\omega=\bar{R}_{2} R_{1} R_{3} e_{2} R_{1} R_{3} \bar{R}_{2}$ belongs to the braid monoid $\widetilde{B}_{4} . \mathbb{P}(\omega)$ denotes the plat closure of $\omega$ that is obtained by attaching maxima at the top and minima at the bottom of the tangle that represents $\omega$.

Note that if $\omega \in \widetilde{B}_{2 n}$, then

$$
\mathbb{P}(\omega) e_{1} e_{3} \ldots e_{2 n-1}=e_{1} e_{3} \ldots e_{2 n-1} \omega e_{1} e_{3} \ldots e_{2 n-1}
$$

in the sense shown below

$$
\mathrm{e}_{1} \mathrm{e}_{3} \omega \mathrm{e}_{1} \mathrm{e}_{3}=
$$

With this much formalism in mind, we can illustrate the important fact that any link diagram can be rearranged via planar isotopy so that it is a plat closure of a word in $\widetilde{B}_{n}$. What we do to obtain this form is make sure that all maxima and minima internal to the diagram are paired in the form $\breve{n}$. Thus if a maximum is not paired to a minimum and there is no arc of the link diagram above it, then this maxima will be one of the top maxima in the plat. If the maximum has an arc 
above it, then we bend this arc to create a minimum to pair with the given maximum:

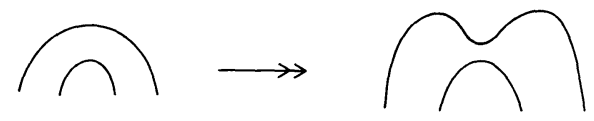

Such a bend may create further maxima. Repeat the procedure for these and do the analog for the minima. The result is a link diagram in plat form, with all internal maxima and minima paired.

Example.

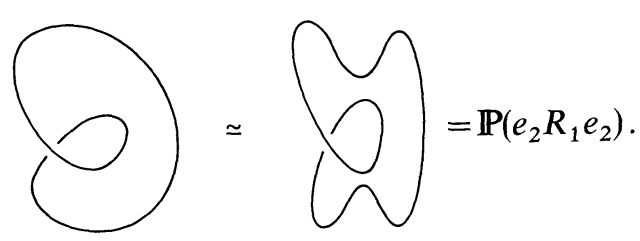

We can now calculate the bracket polynomial for any plat by the procedure of substitution:

$$
\begin{aligned}
& R_{i}=A e_{i}+B \leftrightarrow[\chi]=A[\mathrm{n}]+B[)(], \\
& \bar{R}_{i}=A+B e_{i} \leftrightarrow[X]=A[)(]+B[\tilde{n}] .
\end{aligned}
$$

Multiply the words formally to obtain an element in the Temperley-Lieb algebra. To calculate the bracket, replace each product of $e_{i}$ 's by its plat trace obtained by closing the product with the plat maxima and minima and taking $d$ raised to the number of loops (this amounts to the Jones trace definition in Proposition 3.3):

$$
\left[\mathbb{P}\left(e_{1} e_{2}\right)\right] e\left(\text { in } \widetilde{B}_{4}\right)=d^{2} \text {. }
$$

The product of $R_{i}, \bar{R}_{i}, e_{i}$ with this substitution will still be denoted by $\omega$.

We can now specialize the discussion to the case of the 4-color problem. With $Q=4$ we have $Q^{1 / 2}=2$, and with $\varepsilon=0$ (for proper colorings) we obtain

$$
A=-\frac{1}{2}, \quad B=1, \quad d=Q^{1 / 2}=2 .
$$

Thus

$$
R_{i}=1-\frac{e_{i}}{2}, \quad \bar{R}_{i}=-\frac{1}{2}+e_{i}, \quad e_{i}^{2}=2 e_{i} .
$$

As we have discussed in Sect. 6 (see also the proof of Theorem 6.4 in the present section), the faithfulness of the trace allows us to conclude that a plane graph $G$ is 4-colorable if and only if the word $\omega=\omega[K(G)]$ (obtained by the plat procedure and the above situations) is non-zero in the Temperley-Lieb algebra.

At this point, we need to characterize the sorts of words that arise from a plane graph. The following lemma is the first ingredient:

Lemma 7.2. Let $G$ be a plane graph, and $\omega=\omega[K(G)]$ a word in the $e_{i}, R_{j}, \bar{R}_{k}$, defined by the plat procedure, then all the indices $i$ for $R_{i}$ appearing in $\omega$ have the same parity, and all occurrences of $\bar{R}_{j}$ have the opposite parity. 
Proof. The lemma follows easily from the fact that the link diagram $K(G)$ is alternating. Using this fact we can trace the connection between the local diagram for $R_{i}$ and the nearest local diagram for $\bar{R}_{j}$ to which it is connected. See the figure below:

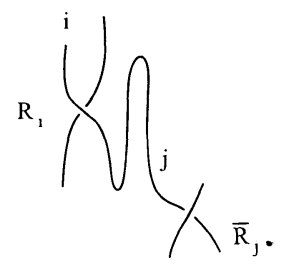

This diagram illustrates schematically the case of a subword of the form $R_{i} W \bar{R}_{j}$, where $W$ is a product of $e_{k}^{\prime}$ 's. For example, $R_{1} e_{3} e_{2} \bar{R}_{4}$ is shown below:

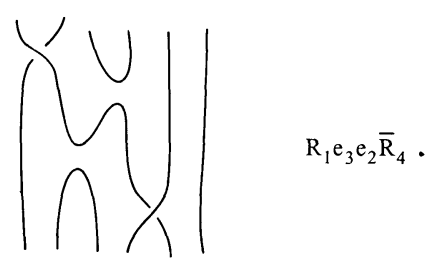

Because the connecting line from $R_{i}$ to $\bar{R}_{j}$ proceeds from strand to strand, and because there must be an even number of intervening strands, we conclude that $i$ and $j$ have opposite parity.

There are other cases to check in completing the proof of this lemma. We will leave these to the reader. They are: 1) the case $R_{i} W \bar{R}_{j}$, where there is no direct connecting arc between $R_{i}$ and $R_{j}$. 2) The case where the truncated link diagram has separated components. The same analysis suffices for these cases, keeping in mind that the graph $G$ corresponds to a checkerboard shading of the link diagram. This completes the proof of the lemma.

Remark. Here is an example that illustrates the omitted cases in the lemma.

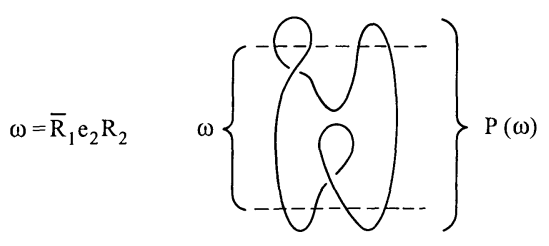

Here $\bar{R}_{1}$ is not directly connected to $R_{2}$, but in the plat closure they are connected and the diagram is alternating.

With these examples in mind, recall that the four color theorem asserts that a plane graph $G$ is colorable with four colors (proper vertex coloration) if and only if $G$ has no loops. Thus, we must examine the algebraic version of a loop via the medial graph translation. The corresponding link diagram is shown below.

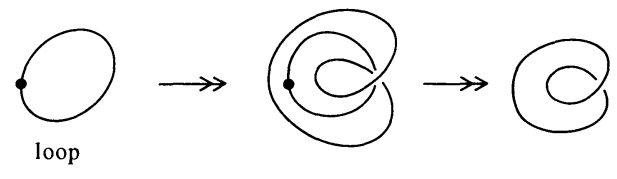


This alternating medial loop configures as a plat in two basic forms:

1.

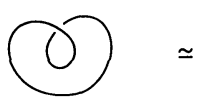

$\Rightarrow \omega=\mathrm{R}_{2} \mathrm{e}_{2}$

2.

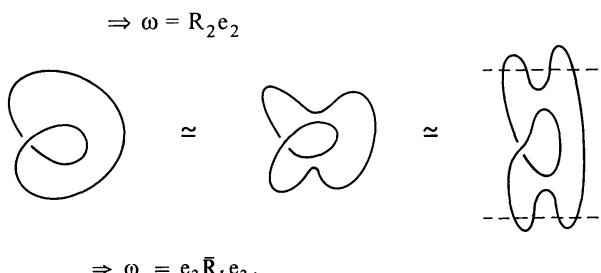

Thus in general, the elements $R_{i} e_{i}, e_{i} R_{i}$, and $e_{i \pm 1} \bar{R}_{i} e_{i \pm 1}$ correspond to loops in the graph $G$. Each of these products is zero in the Temperley-Lieb algebra:

$$
\begin{aligned}
R_{i} e_{i} & =\left(1-\frac{e_{i}}{2}\right) e_{i}=e_{i}-\frac{e_{i}^{2}}{2}=e_{i}-e_{i}=0 \\
e_{i+1} \bar{R}_{i} e_{i+1} & =e_{i+1}\left(-\frac{1}{2}+e_{i}\right) e_{i+1}=e_{i+1} e_{i} e_{i+1}-\frac{e_{i+1}^{2}}{2} \\
& =e_{i+1}-e_{i+1}=0 .
\end{aligned}
$$

Therefore, say that a word $\omega$ in $e_{i}, R_{j}, \bar{R}_{k}$ is loop-free if up to exterior equivalence (Definition 6.3) there is no appearance of an element of the set $\left\{e_{i} R_{i}, e_{i} \bar{R}_{i \pm 1} e_{i}\right\}$ as a subword of $\omega$.

Call a word $\omega$ graphic if all indices among the $R_{i}$ have the same parity, and all indices among the $\bar{R}_{j}$ have the opposite parity.

With this terminology, we recover the two results of Sect. 6:

Theorem 6.4. Let $\omega$ be loop-free and graphic (in the Temperley-Lieb algebra with loop value 2 as described above), and let $G$ be any plane graph such that $\omega=\omega(G)$. Then $\omega \neq 0$ in the Temperley-Lieb algebra if and only if $G$ is 4-colorable. Hence, by the four-color theorem it follows that $\omega \neq 0$.

Conjecture 1. If $\omega$ is loop-free then $\omega \neq 0$.

Theorem 6.4 is equivalent to the four-color theorem. Conjecture 1 is a generalization of the four-color theorem. From our algebraic stance, both appear equally likely.

Proof of Theorem 6.4. By Lemma 7.2 and the hypothesis that $\omega$ is loop-free and graphic, it follows that there is a plane graph $G$ such that $\omega=\omega(G)$ and that $G$ is loop-free as a planar graph. We have established that the number of proper 4-colorings of $G$ is given by the bracket evaluation $2^{V_{(G)}}\{\mathbb{P}(\omega)\}$, where $\mathbb{P}$ denotes the plat closure of $\omega$, and $V_{G}$ is the number of vertices in the graph $G$. Note that $\mathbb{P}(\omega)=\omega * e_{1} e_{3} \ldots e_{2 k-1}$ for some $k$ a positive integer, where $a * b$ denotes the result of attaching top to top and bottom to bottom the tangles associated with the Temperley-Lieb elements $a$ and $b$. If $\tau$ is any other word in $e_{1}, \ldots, e_{2 k-1}$ (even indices included) then $\omega * \tau$ is the medial link diagram of a graph obtained from $G$ 
by identifying some of its vertices with each other. Thus if $G$ is an uncolorable graph, then it follows that $\{\omega * \tau\}=0$ for all $\tau$ in the Temperley-Lieb algebra. Faithfulness of the Jones-trace is equivalent to the statement that $\{\omega * \tau\}=0$ for all $\tau$ implies that $\omega=0$. Thus, we conclude that if $G$ is uncolorable, then $\omega=0$. Therefore, $G$ is uncolorable if and only if $\omega=0$. Therefore, $G$ is 4-colorable if and only if $\omega \neq 0$. This completes the proof of Theorem 6.4.

\section{The Beraha Conjecture}

For $Q \geqq 4$ it is known that the Jones trace (Proposition 3.3) is faithful. We can thus reformulate the 4 color result and the results of Sect. 5 in the following observation.

Fact 8.1. $Q$ being an integer

- if the Jones trace on the corresponding Temperley-Lieb algebra is faithful then all planar graphs (without loops) are $Q$ colorable;

- if the Jones trace is not faithful, there are planar graphs (without loops) that are not $Q$ colorable. For one of them, the transfer matrix coincides with the symmetrizer that acquires a vanishing Jones trace for this value of $Q$.

For $Q$ not an integer, one can still consider the properties of the chromatic polynomials, which can be calculated through Proposition 1.3. Of special interest are the real zeroes of $P_{G}(Q)$. Fact 8.1 suggests they might have something to do with the faithfulness of the Jones trace. Let us discuss this question.

The Jones trace is not faithful for $Q=4 \cos ^{2} \frac{\pi p}{n}$ with $\operatorname{gcd}(p, n)=1, n \geqq 2$. A special subset of these values is given by $Q=B_{n}$ where

Definition 8.2. The Beraha numbers [Be] are $B_{n}=4 \cos ^{2} \frac{\pi}{n}, n \geqq 2$.

One has $B_{2}=0, B_{3}=1, B_{4}=2$. Except for these values, it seems the $B_{n}$ 's cannot be zeroes of chromatic polynomials for finite graphs. However, numerical evidence suggests there are zeroes in an asymptotic sense. More precisely, one has the Beraha conjecture $[\mathrm{Be}, \mathrm{Ba}]$.

Conjecture 2. Consider a family of planar graphs $G_{i}$ (without loops) with $V_{G_{i}} \rightarrow \infty$ as $i \rightarrow \infty$. Then the only possible limit points of real zeroes of the chromatic polynomials $P_{G_{i}}(Q)$ are the Beraha numbers.

Notice it may well be that the real zeroes of the chromatic polynomials $P_{G_{i}}(Q)$ have no limits as $i \rightarrow \infty$. The conjecture just states that if they do, then these limits belong to the Beraha numbers.

The "numerical" coincidence between the appearance of the Beraha numbers in this conjecture and in the non-faithfulness of the Jones trace is striking, and goes in the direction of Fact 8.1. A heuristic explanation of the Beraha conjecture has actually been given in [Sa] using the transfer matrix approach and properties of the Temperley-Lieb algebra. The work $[\mathrm{Sa}]$ is based on physical ideas, and its results do not have a rigorous status yet. It suggests, however, that Conjecture 2 is right, and that both the 4 color theorem and the Beraha conjecture have the same algebraic origin. 


\section{Appendix on Reformulations of the Four Color Problem}

The purpose of this appendix is to recall some facts, history, and other points of view about reformulations of the four color problem.

The original form of the problem was coloring the regions of a plane map so that no two regions have the same color. Kempe [Ke] observed that it was sufficient to color maps such that exactly three countries meet at a given vertex. His observation depends upon the addition of a new country at any non-trivalent vertex, as shown below:

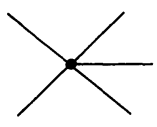

old

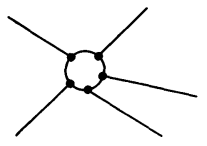

new.

In the new map, every vertex is trivalent, and any coloring of the new map gives a coloring of the old map by simply collapsing the new country to a point.

Tait $[\mathrm{Ta}]$ showed that coloring the map with four colors on its faces is equivalent to coloring the edges with three colors - so that three distinct colors meet at each vertex. (The map is now assumed to be trivalent.) This reformulation is accomplished by regarding the four colors as elements of the group $\mathbb{Z}_{2} \times \mathbb{Z}_{2}$ with generators (say) R, B, P (red, blue, purple), and W (white) the identity element. Thus $\mathrm{RB}=\mathrm{BR}=\mathrm{P}, \mathrm{R}^{2}=\mathrm{B}^{2}=\mathrm{P}^{2}=\mathrm{W}$ and colors commute. Then, given a coloring of the map, we color each edge with the product of the colors on its adjoining faces. This gives the desired 3-coloring of the trivalent edge graph.

Tait's reformulation in terms of the edge coloring was quite significant for a number of reasons. First of all, it led to a further reformulation by Heawood [He] in terms of signs $( \pm 1)$ attached to the vertices of the map. Assign to each vertex of a tricolored trivalent graph in the plane +1 or -1 according to the scheme below:
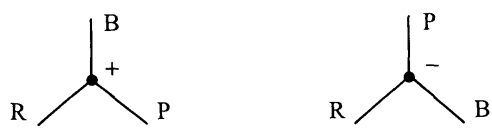

That is, we choose the clockwise cyclic order (RBP) as + and the anticlockwise cyclic order (RPB) as -. Heawood then discovered that with this assignment of \pm 1 to each vertex, the sum of the signs around any face of the map is congruent to zero modulo 3. For example,

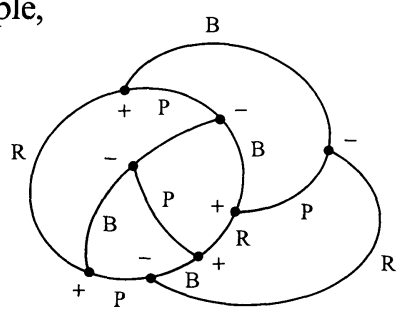

This led Heawood to his famous near-algebraic reformulation obtained by writing down the system of equations associated with a given map. One variable is assigned to each vertex, one equation to each face [the sum of the vertices around 
each face is set equal to zero $(\bmod 3)]$. Any solution to this algebra problem gives a coloring of the original plane map. Heawood's reformulation is not quite a complete translation into algebra since the set of equations must be retrieved from the drawing of a given plane map.

Many other reformulations of the four-color theorem could be mentioned here. There are two more modern ones that we will indicate since they are related to Lie algebras, statistical mechanics and Heawood's original ideas. First, the statistical mechanics: In $[\mathrm{Pe}]$, Penrose gives the following prescription for finding the number of edge tricolorings of a plane trivalent map: assign to each vertex of a given tricoloring $\pm \sqrt{-1}$, where the sign \pm 1 is the Heawood sign of the vertex. If $\sigma$ is a coloring of $G$, let $\|\sigma\|$ denote the product of these $+\sqrt{-1}$ and $-\sqrt{-1}$ over the vertices of the coloring.

If $G$ is not embedded in the plane, it may be immersed with singular edges as in

G

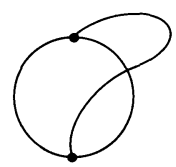

(That is, the singularities of the embedding consist of transversal intersections of edges of the graph at interior points of the edges.) We can still consider colorings of $G$, and since each vertex receives three colors in a cyclic order determined by the planar embedding of that vertex, we can still assign $\pm \sqrt{-1}$ to each vertex. Define $\|\sigma\|$ as before.

Now for $G$ an immersed trivalent plane graph, let $\|G\|=\sum_{G}\|\sigma\|$, where $\sigma$ runs over all edge three-colorings of $G$ with three colors incident to each vertex.

Penrose observes that this partition function, $\|G\|$, is equal to the number of colorings of $G$ when $G$ is embedded in the plane, and that $\|G\|$ satisfies the recursion formulas:

$$
\begin{aligned}
& \|Y\|=\|)(\|-\| X \| \\
& \|O\|=3
\end{aligned}
$$

For example,

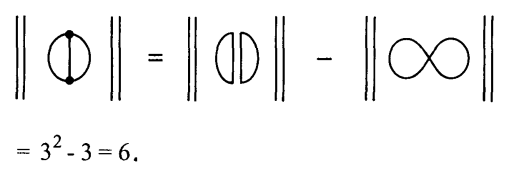

The Penrose formula shows how the coloring problem is related to another sort of statistical sum, this time related to spin networks and the theory of angular momentum.

Finally, we mention the algebraic reformulation of the four color theorem that is proved in [Ka3]. Let $V$ denote the vector cross product algebra on threedimensional space. That is, $V=\{a i+b j+c k\}$, where $a, b, c$ are real numbers, $\{i, j, k\}$ is the usual vector basis for $\mathbb{R}^{3}$, and $i^{2}=j^{2}=k^{2}=0 ; i j=k, j i=-k ; j k=i, k j=-i$; $k i=j, i k=-j$. The algebra $V$ is non-associative - for example $(i i) j=0$ while $i(i j)=i k=-j$. 
Consider the following problem: Let $\mathscr{L}$ and $\mathscr{R}$ denote two associations of the product $X_{1} X_{2} X_{3} \ldots X_{n}$. [An association is an assignment of parentheses to the product so that it is seen as a sequence of binary products. Thus $\left(X_{1} X_{2}\right)\left(X_{3} X_{4}\right)$ and $X_{1}\left(X_{2}\left(X_{3} X_{4}\right)\right)$ are two associations of $X_{1} X_{2} X_{3} X_{4}$.]

Cross Product Problem. Solve the equation $\mathscr{L}=\mathscr{R}$ by assigning to each $X$ a value equal to $i$ or $j$ or $k$ so that $\mathscr{L}=\mathscr{R} \neq 0$. [For example, $(k i)(j i)=k(i(j i))$ and both sides equal $(-i) \neq 0$.]

In [Ka 3] it is shown that the four color theorem is equivalent to the solvability of the Cross Product Problem for every $n>1$ and every choice of parenthesizations $\mathscr{L}$ and $\mathscr{R}$. As explained in [Ka 3], this problem has common roots with the Penrose recursion. Hence, the four color theorem is seen as a subtle property of the Lie algebra of $S O(3)$ (i.e. the vector cross product algebra).

The vector cross product reformulation and the Temperley-Lieb reformulation are the two known fully algebraic reformulations of the four color problem. One intriguing line for further research is to better understand the relationship between these two points of view.

Acknowledgements. H.S. thanks the Enrico Fermi Institute, where this work was started, for its hospitality. He also thanks I. Frenkel for several discussions and encouragement. L.K. thanks M.S.R.I., Berkeley, and R.I.M.S., Kyoto, for their hospitality during the preparation of this work.

\section{References}

[AH] Appel, K.I., Haken, W.: Every planar map is four colorable. Bull. Am. Math. Soc. 82, 711 (1976)

[Ba] Baxter, R.J.: Exactly solved models in statistical mechanics. New York: Academic Press, 1982

[Be] Beraha, S.: Infinite non-trivial families of maps and chromials. Thesis, Johns Hopkins University

Beraha, S., Kahane, J., Weiss, N.J.: J. Comb. Theory B 28, 52 (1980)

[D] Dieudonne, J.: Pour l'honneur de l'esprit humain. Les Mathématiques aujourd'hui. Paris (1991)

[F] Fary, I.: On the straight line representations of planar graphs. Acta. Sci. Math. 11, 229 (1948)

[GHJ] Goodman, F.M., Harpe, P. de la, Jones, V.: Coxeter graphs and towers of algebras. Mathematical Sciences Research Institute Publications 14. Berlin, Heidelberg, New York: Springer, 1989

[He] Heawood, P.J.: On the four colour map theorem. Quart. J. Math. 29, 270 (1898)

[Jo] Jones, V.: Invent. Math. 72, 1 (1983)

[Ka 1] Kauffman, L.H.: State models and the Jones polynomial. Topology 26, 395 (1987)

[Ka 2] Kauffman, L.H.: Statistical mechanics and the Jones polynomial. Proceedings of the 1986 Santa Cruz conference on Artin's Braid Group, A.M.S. Contemp. Math. Series 78, 263 (1989)

[Ka 3] Kauffman, L.H.: Map coloring and the vector cross product. J. Comb. Th. B. 48, 145 (1990)

[Ke] Kempe, A.B.: On the geographical problem of four colors. Am. J. Math. 2, 193 (1879)

[Pe] Penrose, P.: Applications of negative dimensional tensors. In: Combinatorial mathematics and its applications. Welsk, D.J.A. (ed.), New York, London: Academic Press, 1971

[PW] Perk, J., Wu, F.Y.: Non-intersecting string model and graphical approach: equivalence with a Potts model. J. Stat. Phys. 42, 727 (1986)

[Sa] Saleur, H.: Zeroes of chromatic polynomials: a new approach to the Beraha conjecture using quantum groups. Commun. Math. Phys. 132, 657 (1990) 
[SB] Saleur, H., Bauer, M.: On some relations between local height probabilities and conformal invariance. Nucl. Phys. B 320, 591 (1989)

[SK] Saaty, T.L., Kainen, P.C.: The four color problem. New York: McGraw Hill, 1977 (and references therein)

[TL] Temperley, H.N.V., Lieb, E.H.: Relations between the percolation and coloring problem and other graph theoretical problems associated with regular planar lattices. Proc. Roy. Soc. (London) A 322, 151 (1971)

[Ta] Tait, L.G.: Remarks on the coloring of maps. Proc. R. Soc. (Edinburgh) 10, 71 (1980)

[W] Wu, F.Y.: Graph theory in statistical mechanics. In: Studies in foundations and combinations. Adv. in Math. Supp. Studies 1, 151 (1978)

[We] Wenzl, H.: Invent. Math. 92, 349 (1988)

Communicated by N. Yu. Reshetikhin 